\title{
Association of adipose tissue and skeletal muscle metrics with overall survival and postoperative complications in soft tissue sarcoma patients: an opportunistic study using computed tomography
}

\author{
Robert D. Boutin ${ }^{1}$, Jeremy R. Katz ${ }^{2}$, Abhijit J. Chaudhari ${ }^{3}$, Jonathan G. Yabes ${ }^{4}$, Jonah S. Hirschbein ${ }^{5}$, \\ Yves-Paul Nakache ${ }^{6}$, J. Anthony Seibert ${ }^{3}$, Ramit Lamba ${ }^{3}$, Ghaneh Fananapazir ${ }^{3}$, Robert J. Canter ${ }^{7 \#,}$ \\ Leon Lenchik ${ }^{8 *}$
}

${ }^{1}$ Department of Radiology, Stanford University Medical Center, Stanford, CA, USA; ${ }^{2}$ Integrated Imaging Associates, Oak Lawn, IL, USA; ${ }^{3}$ Department of Radiology, University of California, Davis, School of Medicine, Sacramento, CA, USA; ${ }^{4}$ Department of Biostatistics, University of Pittsburgh, Pittsburgh, PA, USA; ${ }^{5}$ Emeryville Advanced Imaging, Emeryville, CA, USA; ${ }^{6}$ Department of Internal Medicine, Santa Clara Valley Medical Center, San Jose, CA, USA; ${ }^{7}$ Department of Surgery, University of California, Davis, School of Medicine, Sacramento, CA, USA; ${ }^{8}$ Department of Radiology, Wake Forest School of Medicine, Winston-Salem, NC, USA

"These authors contributed equally to this work.

Correspondence to: Robert D. Boutin, MD. Department of Radiology, Stanford University Medical Center, 300 Pasteur Drive (MC5105), Stanford, CA 94305-5105, USA. Email: boutin@stanford.edu.

Background: To determine the relationship between adipose tissue and skeletal muscle measurements on computed tomography (CT) and overall survival and major postoperative complications in patients with softtissue sarcoma (STS).

Methods: The retrospective study included 137 STS patients ( 75 men, 62 women; mean age, 53 years, SD 17.7; mean BMI, 28.5, SD 6.6) who had abdominal CT exams. On a single CT image, at the L4 pedicle level, measurements of visceral adipose tissue (VAT), subcutaneous adipose tissue (SAT), and skeletal muscle area and attenuation were obtained using clinical PACS and specialized segmentation software. Clinical information was recorded, including STS characteristics (size, depth, grade, stage, and site), overall survival, and postoperative complications. The relationships between CT metrics and survival were analyzed using Cox proportional hazard models and those between CT metrics and postoperative complications using logistic regression models.

Results: There were 33 deaths and 41 major postoperative complications. Measured on clinical PACS, the psoas area $(\mathrm{P}=0.003)$, psoas index $(\mathrm{P}=0.006)$, psoas attenuation $(\mathrm{P}=0.011)$, and total muscle attenuation $(\mathrm{P}=0.023)$ were associated with overall survival. Using specialized software, psoas attenuation was also associated with overall survival $(\mathrm{P}=0.018)$. Adipose tissue metrics were not associated with survival or postoperative complications.

Conclusions: In STS patients, CT-derived muscle size and attenuation are associated with overall survival. These prognostic biomarkers can be obtained using specialized segmentation software or routine clinical PACS.

Keywords: Computed tomography (CT); muscle; myosteatosis; sarcopenia; soft-tissue sarcoma (STS)

Submitted Oct 28, 2019. Accepted for publication Feb 04, 2020.

doi: 10.21037/qims.2020.02.09

View this article at: http://dx.doi.org/10.21037/qims.2020.02.09 


\section{Introduction}

The interpretation of CT examinations in patients with neoplasms routinely focuses on documenting the lesion site, size, and extent, as well as providing the differential diagnosis. Although body composition information is available on these CT exams, it is usually not reported.

Body composition can affect cancer development and cancer prognosis (1-4). Obesity, for example, is an important risk factor for developing 10 of 17 common cancers $(5,6)$. CT measures of visceral adipose tissue (VAT) have been associated with survival with some types of cancers (e.g., colorectal and pancreatic cancers), but not with others (e.g., renal cell carcinoma) (4,7-9). In patients with soft-tissue sarcoma (STS), there is currently no compelling evidence about VAT influencing patient survival.

There is also accumulating evidence that skeletal muscle mass and attenuation are associated with health outcomes in many cancers, including those involving the abdominopelvic viscera (1,10-15). In a meta-analysis of 38 studies in 7,843 patients with solid tumors (1), low muscle mass measured on CT was associated with poorer overall survival (HR: 1.44 , 95\% CI: $1.32-1.56, \mathrm{P}<0.001)$. Caution is warranted, however, because prognosis is influenced by many variables, including cancer type. With esophageal cancer, for example, most studies have found no association between CT-derived muscle mass and overall survival (16-20). In patients with STS, two studies seem to show conflicting results regarding the role of CT muscle metrics. In one study (21), low psoas muscle attenuation was associated with higher mortality, but in another study (22) low psoas muscle area showed no association with outcomes.

Although CT measures of adipose tissue and skeletal muscle outperform body mass index (BMI) in risk stratification for many cancers, there are several barriers to clinical implementation, including access to segmentation software used in most research studies (11-15). This specialized software requires considerable time and resources (e.g., data transfer and analysis on an independent workstation). A much easier alternative is to derive these CT metrics on clinical PACS workstations. While VAT and subcutaneous adipose tissue (SAT) measurements on CT are fairly standardized, there is continuing debate on the best methodology to characterize muscle on CT images $(23,24)$. We are not aware of any studies using both specialized segmentation software and routine clinical PACS software to evaluate muscle in patients with STS. To address this gap, our aim was to use specialized and PACS software to investigate adipose tissue and skeletal muscle metrics as prognostic indicators for overall survival and major postoperative complications in STS patients.

\section{Methods}

\section{Patients}

After institutional review board approval with waiver of informed consent for this HIPAA-compliant retrospective study, a database of patients under the care of one subspecialty surgeon (RJC) at our tertiary care academic medical center was accessed. Inclusion criteria were: (I) available clinical data that included patient age at the time of presentation, sex, BMI, hemoglobin, creatinine, and C-reactive protein; (II) available neoplasm characteristics including maximal tumor dimension, depth, grade, stage, site, and histology; and (III) a baseline staging CT examination of the abdomen within three months of diagnosis, as per institutional protocol. This initial survey yielded 148 consecutive patients. Exclusion criteria were: (I) abdominal CT images were not available ( $\mathrm{n}=6$ ) or (II) abdominal CT images were of inadequate quality [e.g., due to tumor involvement $(n=3)$ or postoperative change $(\mathrm{n}=2)]$, as determined by consensus of the two authors (RD Boutin, JR Katz) ( $\mathrm{n}=11)$. This yielded 137 patients for the overall survival analysis. Nine patients were treated nonoperatively, and therefore analysis of major postoperative complications included 128 patients.

Two major clinical outcomes were evaluated: overall survival and major postoperative complications. Overall survival was defined as the time from diagnosis to the time of death or to the study termination date. The latter were subsequently censored to the last date at which patients were documented to be alive. Major postoperative complications were defined as return to the operating room for wound repair, an invasive postoperative procedure (e.g., abscess drainage), hospital readmission for wound care (e.g., intravenous antibiotics), or prolonged wound packing (i.e., greater than 120 days from the date of operation) (25).

\section{CT image analysis}

\section{Segmentation procedure}

All radiologists were blinded to clinical data, including outcomes. The same axial CT image at the L4 pedicle level was used to obtain all fat and muscle measurements. Manual segmentation was performed by three radiologists 
(musculoskeletal fellow, third-year resident, second-year resident) who were trained to segment relevant tissue regions by a senior musculoskeletal radiologist with 20 years of research experience.

\section{Adipose tissue metrics}

VAT and SAT were measured on an independent workstation with a public-domain, Java-based, imageprocessing software (ImageJ, v1.42q; National Institutes of Health, http://rsb.info.nih.gov/ij). This method uses a "thresholding" technique, so that only pixels with CT numbers from -30 to $-190 \mathrm{HU}$ are analyzed as fat $(26,27)$. Using this technique, the following were measured: (I) VAT area $\left(\mathrm{cm}^{2}\right)$; (II) VAT attenuation (HU); (III) SAT area $\left(\mathrm{cm}^{2}\right)$; and (IV) SAT attenuation (HU).

\section{Muscle metrics}

Muscle tissues were analyzed using two image-processing methods: (I) thresholded and (II) non-thresholded.

\section{Thresholded}

This method used the same software as the adipose tissue measurement (ImageJ). For muscle measurement, only pixels from -29 to $150 \mathrm{HU}$ were analyzed for area and attenuation calculations. This method is commonly used in body composition research and is intended to capture only "lean muscle" by excluding intramuscular adipose tissue $(26,27)$.

\section{Non-thresholded}

All muscle measurements were also made without thresholding, by drawing a region of interest around the individual muscles or muscle groups using the clinical PACS software (iSite, version 3.6, Philips Healthcare; Foster City, CA).

Both the thresholded and non-thresholded methods yielded muscle area and muscle attenuation metrics. For the psoas, the following muscle metrics were recorded: (I) psoas area $\left(\mathrm{cm}^{2}\right)$; (II) psoas area normalized for patient height, termed the psoas index $\left(\mathrm{cm}^{2} / \mathrm{m}^{2}\right)$; and (III) psoas attenuation (HU). For the combination of all the psoas, paravertebral, and abdominal musculature, the following muscle metrics were recorded: (I) total muscle area $\left(\mathrm{cm}^{2}\right)$; (II) total muscle index $\left(\mathrm{cm}^{2} / \mathrm{m}^{2}\right)$; and (III) total muscle attenuation (HU).

\section{Statistical analysis}

Descriptive statistics and analyses were obtained using Stata version 12 (StataCorp, College Station, TX). Because of the low number $(\mathrm{n}=12)$ of stage 4 patients, statistical analyses combined stages 3 and 4 . Univariable and multivariable analyses for overall survival were performed using the Cox proportional hazards model to evaluate the covariate effects of each variable. Logistic regression models were used to evaluate the association of CT metrics with major postoperative complications. Age and sex adjustments were used to account for known normative differences in fat and muscle composition in these groups. Significant variables from the multivariable model were analyzed further using multiple hypothesis testing with the Sidak correction. The concordance C-statistic for each model was also calculated. A C-statistic of 0.5 has no discriminating ability for survival versus death, while a value of 1.0 has perfect discrimination. $95 \%$ confidence intervals were calculated. Results were considered significant at $\mathrm{P}<0.05$. Intra-observer and interobserver analysis were performed on a random subset of 14 patients (approximately 10\% of the cohort) and the concordance correlation coefficients were calculated.

\section{Results}

\section{Overall survival}

Patient characteristics are shown in Table 1. Of the 137 patients included in the study, there were 33 (24\%) deaths, with a mean survival time of 33.1 months in those patients. Mean follow-up time for the 104 (76\%) survivors was 28.3 months. There was no significant association of age, sex, ethnicity, hemoglobin, creatinine, C-reactive protein, or tumor size, depth, and site with overall survival. However, there was a significant association of STS grade $(\mathrm{P}=0.001)$ and stage $(\mathrm{P}<0.001)$ with overall survival.

At presentation, patients varied widely in their $\mathrm{BMI}$, adipose tissue, and muscle metrics. BMI had no significant association with overall survival $(\mathrm{P}=0.210)$. Table 2 shows adipose tissue and muscle metrics analyzed using univariable models. Table 3 shows the same CT metrics analyzed using a multivariable Cox proportional hazards model, adjusted for age (dichotomized at age 65), sex, tumor grade, and tumor stage.

\section{Adipose tissue metrics}

Visceral fat, subcutaneous fat, or total fat metrics showed no significant association with overall survival using univariable or multivariable analyses.

\section{Muscle metrics Thresholded}

Using the thresholded (specialized software) method, two metrics were significantly associated with survival: the 
Table 1 Initial clinicopathologic characteristics of soft-tissue sarcoma patients, with univariable analysis for overall survival $\left(\mathrm{n}=137^{\dagger}\right)$

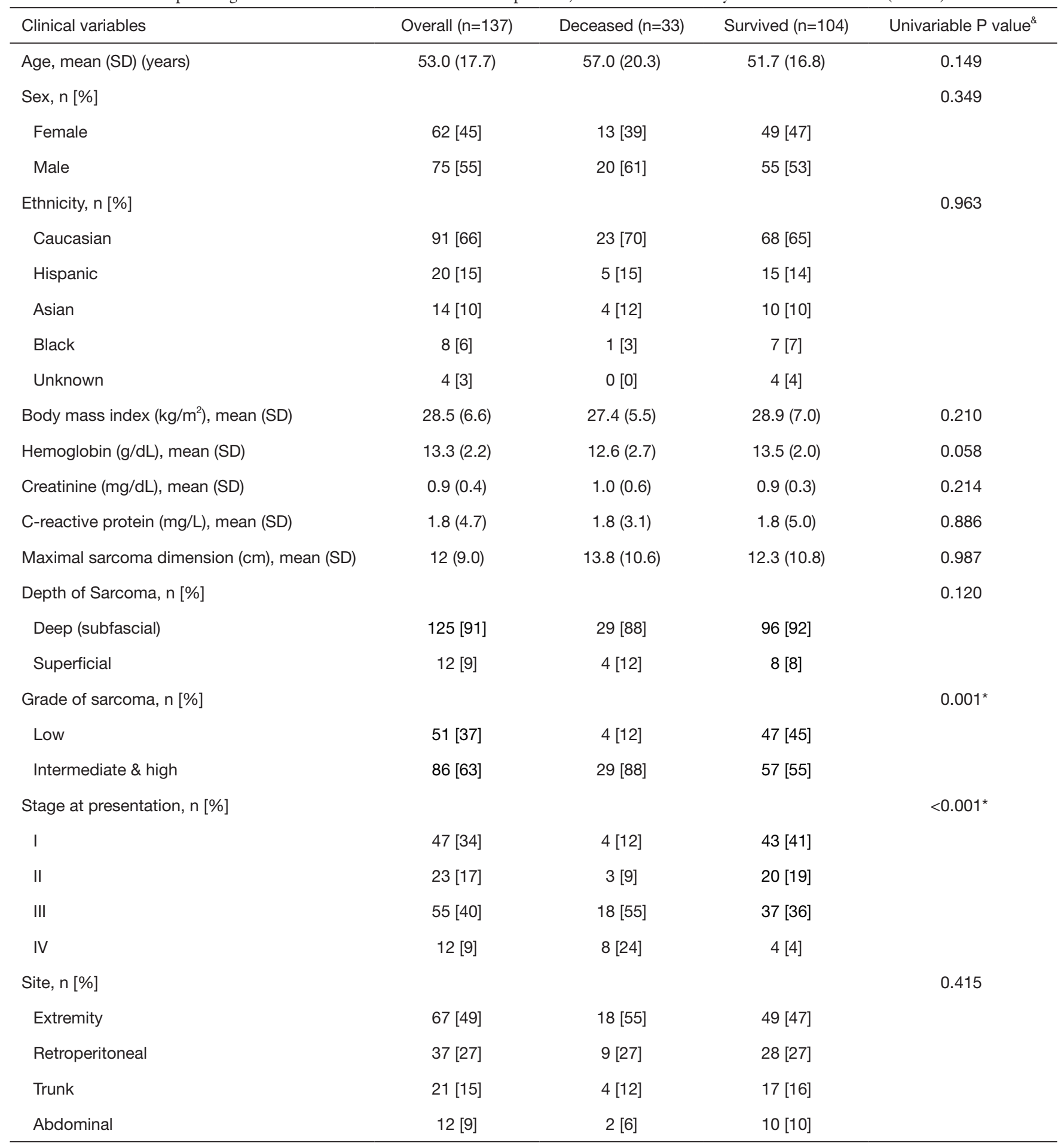

Table 1 (continued) 
Table 1 (continued)

\begin{tabular}{lccc}
\hline Clinical variables & Overall $(n=137)$ & Deceased $(n=33)$ & Survived $(n=104)^{\text {Univariable P value }}$ \\
\hline Histology, $n$ [\%] & $18[13]$ & $7[21]$ & $11[11]$ \\
High-grade UPS & $18[13]$ & $2[6]$ & $16[15]$ \\
Well-differentiated LS & $15[11]$ & $2[6]$ & $13[13]$ \\
Myxoid LS & $11[8]$ & $4[12]$ & $7[7]$ \\
Leiomyosarcoma & $10[7]$ & $1[3]$ & $9[9]$ \\
Dedifferentiated LS & $9[7]$ & $2[6]$ & $7[7]$ \\
Synovial sarcoma & $56[41]$ & $15[45]$ & $41[39]$ \\
Other &
\end{tabular}

Treatments included surgery (87.6\%), as well as radiation therapy before surgery (25.2\%), pre-operative chemotherapy (8.7\%), and administration of a tyrosine kinase inhibitor (1.6\%). ${ }^{\dagger}$, only 128 patients underwent surgical resection with curative intent, of whom 104 were living at last follow up and 24 who died; ${ }^{\text {, }}$, the $\mathrm{P}$ value is based on a Cox proportional hazards univariable model or the log rank test; *, significant values $(\mathrm{P}<0.05)$ are highlighted. UPS, undifferentiated pleomorphic sarcoma; LS, liposarcoma.

Table 2 Adipose tissue and muscle metrics for entire cohort of soft-tissue sarcoma patients, with univariable analysis for overall survival

\begin{tabular}{|c|c|c|c|c|}
\hline Body composition metric & Overall $(n=137)$ & Deceased $(n=33)$ & Survived $(n=104)$ & $P$ value ${ }^{\dagger}$ \\
\hline \multicolumn{5}{|l|}{ Fat, mean (SD) } \\
\hline Visceral fat area & $271.4(132.1)$ & $261.7(126.4)$ & $274.5(134.3)$ & 0.493 \\
\hline Visceral fat attenuation & $-85.9(10.6)$ & $-86.7(10.3)$ & $-85.6(10.7)$ & 0.509 \\
\hline Subcutaneous fat area & $263.2(132.4)$ & $265.2(134.8)$ & $262.5(132.2)$ & 0.942 \\
\hline Total fat area & $712.2(236.3)$ & $695.1(251.5)$ & $717.7(232.3)$ & 0.511 \\
\hline Total fat attenuation & $-90.6(10.2)$ & $-91(10.5)$ & $-90.5(10.1)$ & 0.637 \\
\hline \multicolumn{5}{|l|}{ Thresholded muscle, mean (SD) } \\
\hline Psoas area & $24.3(9.3)$ & $22.6(7.6)$ & $24.9(9.8)$ & 0.288 \\
\hline Total muscle area & $139.9(42.8)$ & $132.5(41.7)$ & $142.2(43.1)$ & 0.548 \\
\hline Total muscle index & $52.3(45.5)$ & $61(90.2)$ & $49.5(12.6)$ & $0.015^{\star}$ \\
\hline Total muscle attenuation & $39(11.8)$ & $36(12.9)$ & $39.9(11.4)$ & 0.088 \\
\hline \multicolumn{5}{|c|}{ Non-thresholded muscle, mean (SD) } \\
\hline Psoas area & $20.5(7.8)$ & $17.6(6.6)$ & $21.4(7.9)$ & $0.019^{*}$ \\
\hline Psoas index & $7.1(2.3)$ & $6.2(1.9)$ & $7.4(2.3)$ & $0.024^{*}$ \\
\hline Psoas attenuation & $50.4(12.6)$ & $46.6(17.6)$ & $51.7(10.3)$ & $0.005^{*}$ \\
\hline Total muscle area & $124.3(42.4)$ & $122.5(41.3)$ & $124.9(42.9)$ & 0.987 \\
\hline
\end{tabular}

\footnotetext{
${ }^{\dagger}$, the $\mathrm{P}$ value is based on a Cox proportional hazards univariable model; ${ }^{*}$, significant values $(\mathrm{P}<0.05)$ are highlighted.
} 
Table 3 Association of CT metrics and overall survival

\begin{tabular}{|c|c|c|c|c|c|c|}
\hline CT metrics & \multicolumn{2}{|c|}{ Univariable analysis $^{\dagger}$} & \multicolumn{2}{|c|}{ Multivariable analysis $^{\ddagger}$} & \multicolumn{2}{|c|}{ Multivariable analysis with Sidak correction ${ }^{\wedge}$} \\
\hline \multicolumn{7}{|l|}{ Adipose tissue } \\
\hline Visceral fat area & 0.999 & 0.493 & 0.998 & 0.234 & - & - \\
\hline Visceral fat attenuation & 0.988 & 0.509 & 0.997 & 0.875 & - & - \\
\hline $\begin{array}{l}\text { Subcutaneous fat } \\
\text { attenuation }\end{array}$ & 0.991 & 0.394 & 0.976 & 0.09 & - & - \\
\hline \multicolumn{7}{|l|}{ Thresholded muscle } \\
\hline Psoas area & 0.977 & 0.288 & 0.942 & 0.078 & $0.928(0.86-1.003)$ & 0.261 \\
\hline Total muscle index & 1.006 & $0.015^{\star}$ & 1.004 & 0.079 & - & - \\
\hline Total muscle attenuation & 0.972 & 0.088 & 0.963 & $0.049^{\star}$ & $0.966(0.929-1.004)$ & 0.342 \\
\hline \multicolumn{7}{|l|}{ Non-thresholded muscle } \\
\hline Psoas area & 0.938 & 0.019 & 0.893 & $<0.001^{*}$ & $0.874(0.81-0.944)$ & $0.003^{*}$ \\
\hline Psoas index & 0.811 & 0.024 & 0.664 & $<0.001^{*}$ & $0.684(0.544-0.86)$ & $0.006^{*}$ \\
\hline Psoas attenuation & 0.969 & 0.005 & 0.963 & $0.002^{\star}$ & $0.963(0.939-0.986)$ & $0.011^{*}$ \\
\hline Total muscle area & 1.000 & 0.987 & 0.996 & 0.411 & - & - \\
\hline
\end{tabular}

${ }^{\dagger}$, the $\mathrm{P}$ value is based on a Cox proportional hazards univariable model; ${ }^{\ddagger}$, the $\mathrm{P}$ value is based on a Cox proportion hazards multivariable model adjusting for age (dichotomized at 65 years), sex, grade, and stage; ${ }^{\wedge}$, the $\mathrm{P}$ value is based on a Cox proportional hazards multivariable model adjusting for age (dichotomized at 65 years), sex, grade, and stage, and it is adjusted using the Sidak correction; *, significant values $(\mathrm{P}<0.05)$ are highlighted.

psoas attenuation $(\mathrm{P}=0.004)$ and total muscle attenuation $(\mathrm{P}=0.049)$. When the Sidak correction to the multivariable model was applied, only psoas attenuation remained significant $(\mathrm{P}=0.018)$, with an adjusted $\mathrm{HR}$ of 0.947 (95\% CI, 0.913-0.982). For every $1 \mathrm{HU}$ increase in the attenuation of the psoas muscle, the hazard of death decreased by $5.3 \%$.

Non-thresholded

Using the non-thresholded (PACS) method, four muscle metrics were significantly associated with mortality: psoas area $(\mathrm{P}<0.001)$, psoas index $(\mathrm{P}<0.001)$, psoas attenuation $(\mathrm{P}=0.002)$, and total muscle attenuation $(\mathrm{P}=0.004)$. With Sidak correction, the adjusted $\mathrm{P}$ values were still significant, ranging from 0.003 to 0.023 . The adjusted C-statistics ranged from 0.78 to 0.81 , demonstrating moderate ability to discriminate between patients who survived and died. With Sidak correction, the HR of psoas attenuation was 0.963 (95\% CI, 0.939-0.986). For every $1 \mathrm{HU}$ increase in attenuation the hazard of death decreased by $3.7 \%$. Similarly, the HR of psoas area was 0.874 (95\% CI, 0.81-0.944). For every $1 \mathrm{~cm}^{2}$ increase in area, the hazard of death decreased by $12.6 \%$.

Total muscle area and total muscle index were not significantly associated with mortality in univariable or multivariable analysis.

\section{Major postoperative complications}

Of 128 patients who had surgery, 41 patients (32\%) had 
Table 4 Association of CT metrics and major postoperative complications

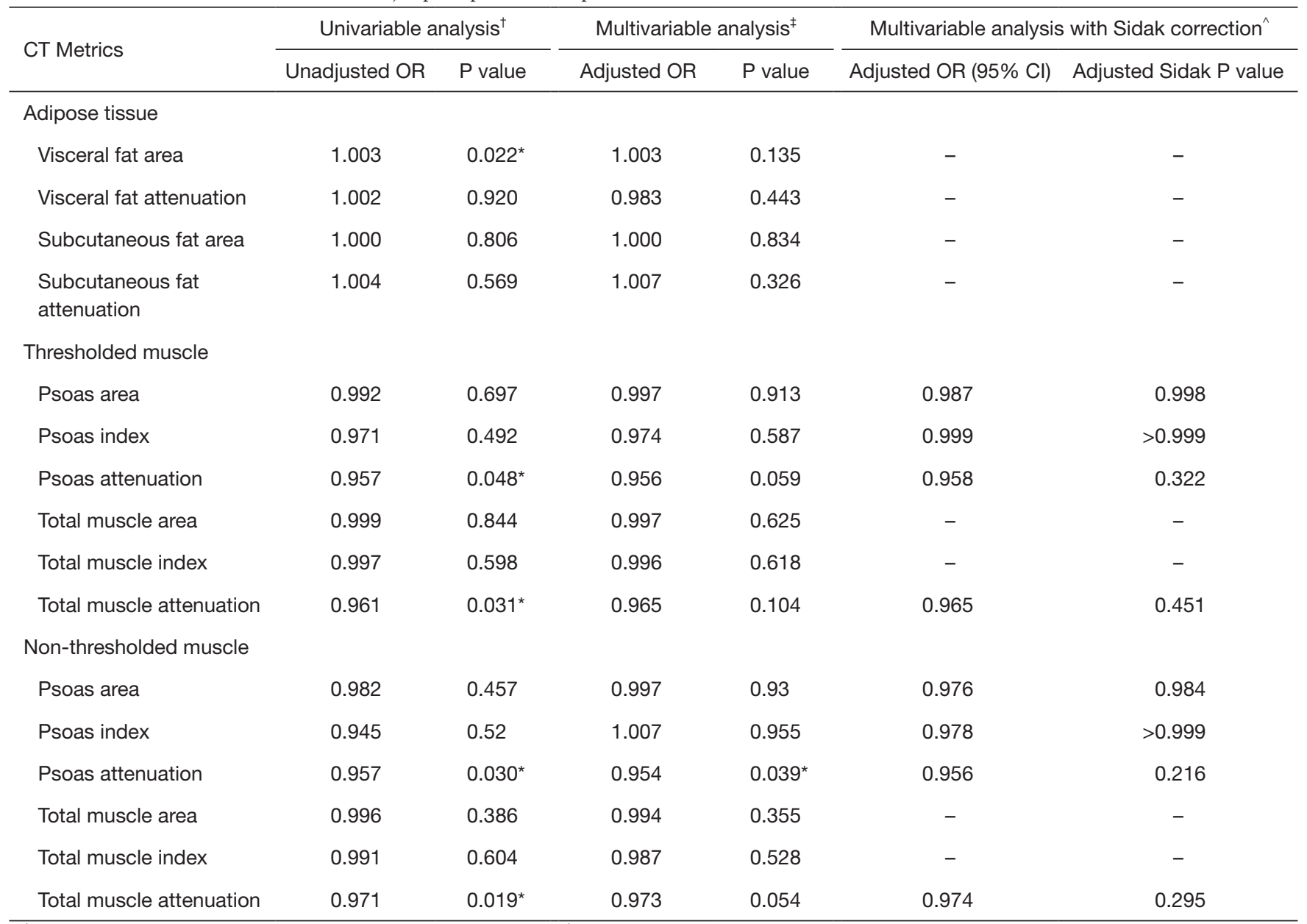

${ }^{\dagger}$, the $\mathrm{P}$ value is based on a logistic univariable model; ${ }^{\ddagger}$, the $\mathrm{P}$ value is based on a logistic multivariable model adjusting for age (dichotomized at 65 years), sex, grade, and stage. ${ }^{\wedge}$, the $\mathrm{P}$ value is based on a logistic multivariable model adjusting for age (dichotomized at 65 years), sex, grade, and stage, and it is adjusted using the Sidak correction; ${ }^{*}$, Significant values $(\mathrm{P}<0.05)$ are highlighted.

a major postoperative complication. Major postoperative complications were more common in patients who died $(9 / 24,38 \%)$ compared to those still living at last follow up $(32 / 104,31 \%)$. BMI had no significant association with major postoperative complications. Table 4 shows univariable and multivariable analyses of major postoperative complications and CT metrics, adjusting for age (dichotomized at age 65), sex, tumor site, and tumor size (Table 4).

\section{Adipose tissue metrics}

Using the thresholded method, 1 of 6 adipose tissue metrics was significant: visceral fat area $(\mathrm{P}=0.022)$. Other adipose tissue metrics were not significantly associated with major postoperative complications.

\section{Muscle metrics}

\section{Thresholded}

Using the thresholded method, 2 of 6 muscle metrics were significantly associated with major postoperative complications in a univariable analyses: psoas attenuation $(\mathrm{P}=0.048)$ and total muscle attenuation (0.031).

\section{Non-thresholded}

Using the non-thresholded method, the same metrics were significant in a univariable analyses: psoas attenuation $(\mathrm{P}=0.030)$ and total muscle attenuation $(\mathrm{P}=0.019)$.

In multivariable analyses, only psoas attenuation remained significant $(\mathrm{P}=0.039$, C-statistic $=0.70)$, but with Sidak correction it was no longer significant $(\mathrm{P}=0.216)$. None of the other muscle metrics (thresholded and nonthresholded) were significant. 


\section{Concordance analysis}

There was excellent intra-observer and inter-observer agreement among the three readers: all correlation coefficients were $>0.9$ for all variables and methods, with one exception. A single inter-observer coefficient (0.74) for the thresholded method of SAT attenuation was attributed to SAT attenuation measurement on a single patient performed by one reader.

\section{Discussion}

Our study shows that shorter survival is significantly associated with low CT measures of psoas muscle area, psoas index, and psoas attenuation in STS patients. These findings remained significant independent of commonly used prognostic factors in patients with cancer, including patient age and BMI.

Prior studies of patients with STS have also analyzed the relationship between CT-derived muscle metrics and survival. Our results on muscle attenuation compare favorably to Veld et al. (21) who studied 116 patients with extremity STS, and reported that psoas muscle attenuation at the L4 level was associated with mortality in fully adjusted models. However, unlike our study, they did not evaluate muscle cross-sectional area or muscle index (21).

Different results were reported by Wilson et al. (22) in 137 patients with STS undergoing a preoperative staging abdominal CT. They used a free-hand (non-thresholded) technique to measure the psoas area at the L3 level (indexed for patient height), and found no association with overall survival or wound complications. In contrast, our study found that a higher psoas index was associated with longer survival, even after adjusting for covariates. This difference may be related to differences in their study population compared to ours: percentage of superficial sarcomas $(20 \%$ vs. $9 \%$ ), undifferentiated pleomorphic sarcoma ( $42 \%$ vs. $13 \%)$, and intermediate/high grade sarcoma ( $82 \%$ vs. $63 \%$ ).

In a prior study of adipose tissue metrics in patients with STS, Veld et al. (21) reported higher attenuation of SAT (but not VAT) was associated with mortality. In our study, we found no association between VAT and SAT metrics and mortality. Another study (28) of 60 patients with extremity STS undergoing non-contrast CTs reported that the attenuation of SAT at the L4 level was associated with postoperative wound infections. This is different from our study, where VAT area was associated with major postoperative complications in unadjusted models, but not significant after adjusting for covariates. Thus, in our cohort, the combination of four covariates (age, sex, grade, and stage) was a stronger determinant of clinical outcomes than VAT cross-sectional area.

A prospective, multi-institutional study is needed to reconcile the differences among STS studies and determine the value of CT-derived adipose and muscle metrics for patient management and prognosis.

Our study has several limitations that are similar to prior single-center studies, including retrospective study design, lack of non-cancer control subjects, heterogeneous histologic subtypes of sarcomas included in analyses, and lack of histologic correlation for the muscle and fat measurements made by CT. However, our study also has several strengths, including the quantitative evaluation of both cross-sectional area and attenuation metrics for adipose tissue and skeletal muscle, with rigorous statistical analysis of important clinical outcomes: overall survival and major postoperative complications.

\section{Conclusions}

In conclusion, muscle area and attenuation are associated with overall survival in patients with STS. These prognostic biomarkers can be quantitated on independent workstations that use thresholding software or measured using routine clinical PACS.

\section{Acknowledgments}

The authors thank Ellen Cheang, MD for helping to process CT data and Patrice Gibbs for helping to process the statistical data.

Funding: The study was supported by National Institutes of Health grant R03 EB015099.

\section{Footnote}

Provenance and Peer Review: With the arrangement by the Guest Editors and the editorial office, this article has been reviewed by external peers.

Conflicts of Interest: All authors have completed the ICMJE uniform disclosure form (available at http://dx.doi. org/10.21037/qims.2020.02.09). The special issue "Body Composition Imaging" was commissioned by the editorial office without any funding or sponsorship. The authors have no other conflicts of interest to declare. 
Ethical Statement: The study was conducted in accordance with the Declaration of Helsinki (as revised in 2013). This study obtained ethics approval from our Institutional Review Board, with waiver of informed consent for this retrospective study.

Open Access Statement: This is an Open Access article distributed in accordance with the Creative Commons Attribution-NonCommercial-NoDerivs 4.0 International License (CC BY-NC-ND 4.0), which permits the noncommercial replication and distribution of the article with the strict proviso that no changes or edits are made and the original work is properly cited (including links to both the formal publication through the relevant DOI and the license). See: https://creativecommons.org/licenses/by-nc-nd/4.0/.

\section{References}

1. Shachar SS, Williams GR, Muss HB, Nishijima TF. Prognostic value of sarcopenia in adults with solid tumours: A meta-analysis and systematic review. Eur J Cancer 2016;57:58-67.

2. Levolger S, van Vugt JL, de Bruin RW, IJzermans JN. Systematic review of sarcopenia in patients operated on for gastrointestinal and hepatopancreatobiliary malignancies. Br J Surg 2015;102:1448-58.

3. Gibson DJ, Burden ST, Strauss BJ, Todd C, Lal S. The role of computed tomography in evaluating body composition and the influence of reduced muscle mass on clinical outcome in abdominal malignancy: a systematic review. Eur J Clin Nutr 2015;69:1079-86.

4. Malietzis G, Aziz O, Bagnall NM, Johns N, Fearon KC, Jenkins JT. The role of body composition evaluation by computerized tomography in determining colorectal cancer treatment outcomes: a systematic review. Eur J Surg Oncol 2015;41:186-96.

5. Bhaskaran K, Douglas I, Forbes H, dos-Santos-Silva I, Leon DA, Smeeth L. Body-mass index and risk of 22 specific cancers: a population-based cohort study of 5.24 million UK adults. Lancet 2014;384:755-65.

6. Lauby-Secretan B, Scoccianti C, Loomis D, Grosse Y, Bianchini F, Straif K; International Agency for Research on Cancer Handbook Working Group. Body Fatness and Cancer--Viewpoint of the IARC Working Group. N Engl J Med 2016;375:794-8.

7. Xiao J, Mazurak VC, Olobatuyi TA, Caan BJ, Prado CM. Visceral adiposity and cancer survival: a review of imaging studies. Eur J Cancer Care (Engl) 2018;27:e12611.
8. Lee CS, Murphy DJ, McMahon C, Nolan B, Cullen G, Mulcahy H, Sheahan K, Barnes E, Fennelly D, Ryan EJ, Doherty GA. Visceral Adiposity is a Risk Factor for Poor Prognosis in Colorectal Cancer Patients Receiving Adjuvant Chemotherapy. J Gastrointest Cancer 2015;46:243-50.

9. Cakir H, Heus C, van der Ploeg TJ, Houdijk AP. Visceral obesity determined by CT scan and outcomes after colorectal surgery; a systematic review and meta-analysis. Int J Colorectal Dis 2015;30:875-82.

10. Boutin RD, Yao L, Canter RJ, Lenchik L. Sarcopenia: Current Concepts and Imaging Implications. AJR Am J Roentgenol 2015;205:W255-66.

11. Martin L, Gioulbasanis I, Senesse P, Baracos VE. CancerAssociated Malnutrition and CT-Defined Sarcopenia and Myosteatosis Are Endemic in Overweight and Obese Patients. JPEN J Parenter Enteral Nutr 2020;44:227-38.

12. Hopkins JJ, Reif RL, Bigam DL, Baracos VE, Eurich DT, Sawyer MB. The Impact of Muscle and Adipose Tissue on Long-term Survival in Patients With Stage I to III Colorectal Cancer. Dis Colon Rectum 2019;62:549-60.

13. Stretch C, Aubin JM, Mickiewicz B, Leugner D, AlManasra T, Tobola E, Salazar S, Sutherland FR, Ball CG, Dixon E, Vogel HJ, Damaraju S, Baracos VE, Bathe OF. Sarcopenia and myosteatosis are accompanied by distinct biological profiles in patients with pancreatic and periampullary adenocarcinomas. PLoS One 2018;13:e0196235.

14. Kroenke CH, Prado CM, Meyerhardt JA, Weltzien EK, Xiao J, Cespedes Feliciano EM, Caan BJ. Muscle radiodensity and mortality in patients with colorectal cancer. Cancer 2018;124:3008-3015.

15. Martin L, Hopkins J, Malietzis G, Jenkins JT, Sawyer MB, Brisebois R, MacLean A, Nelson G, Gramlich L, Baracos VE. Assessment of Computed Tomography (CT)-Defined Muscle and Adipose Tissue Features in Relation to ShortTerm Outcomes After Elective Surgery for Colorectal Cancer: A Multicenter Approach. Ann Surg Oncol 2018;25:2669-2680.

16. Grotenhuis BA, Shapiro J, van Adrichem S, de Vries M, Koek M, Wijnhoven BP, van Lanschot JJ. Sarcopenia/ Muscle Mass is not a Prognostic Factor for Short- and Long-Term Outcome After Esophagectomy for Cancer. World J Surg 2016;40:2698-704.

17. Reisinger KW, Bosmans JW, Uittenbogaart M, Alsoumali A, Poeze M, Sosef MN, Derikx JP. Loss of Skeletal Muscle Mass During Neoadjuvant Chemoradiotherapy Predicts Postoperative Mortality in Esophageal Cancer Surgery. 
Ann Surg Oncol 2015;22:4445-52.

18. Elliott JA, Doyle SL, Murphy CF, King S, Guinan EM, Beddy P, Ravi N, Reynolds JV. Sarcopenia: Prevalence, and Impact on Operative and Oncologic Outcomes in the Multimodal Management of Locally Advanced Esophageal Cancer. Ann Surg 2017;266:822-30.

19. Panje CM, Hong L, Hayoz S, Baracos VE, Herrmann E, Garcia Schuler H, Meier UR, Henke G, Schacher S, Hawle H, Gérard MA. Skeletal muscle mass correlates with increased toxicity during neoadjuvant radiochemotherapy in locally advanced esophageal cancer: A SAKK 75/08 substudy. Radiat Oncol 2019;14:166.

20. Murimwa GZ, Venkat PS, Jin W, Leuthold S, Latifi K, Almhanna K, Pimiento JM, Fontaine JP, Hoffe SE, Frakes JM. Impact of sarcopenia on outcomes of locally advanced esophageal cancer patients treated with neoadjuvant chemoradiation followed by surgery. J Gastrointest Oncol 2017;8:808-15.

21. Veld J, Vossen JA, De Amorim Bernstein K, Halpern EF, Torriani M, Bredella MA. Adipose tissue and muscle attenuation as novel biomarkers predicting mortality in patients with extremity sarcomas. Eur Radiol 2016;26:4649-4655.

22. Wilson RJ, Alamanda VK, Hartley KG, Mesko NW, Halpern JL, Schwartz HS, Holt GE. Sarcopenia Does Not Affect Survival or Outcomes in Soft-Tissue Sarcoma. Sarcoma 2015;2015:146481.

23. Hopkins JJ, Skubleny D, Bigam DL, Baracos VE,

Cite this article as: Boutin RD, Katz JR, Chaudhari AJ, Yabes JG, Hirschbein JS, Nakache YP, Seibert JA, Lamba R, Fananapazir G, Canter RJ, Lenchik L. Association of adipose tissue and skeletal muscle metrics with overall survival and postoperative complications in soft tissue sarcoma patients: an opportunistic study using computed tomography. Quant Imaging Med Surg 2020;10(8):1580-1589. doi: 10.21037/ qims.2020.02.09
Eurich DT, Sawyer MB. Barriers to the Interpretation of Body Composition in Colorectal Cancer: A Review of the Methodological Inconsistency and Complexity of the CT-Defined Body Habitus. Ann Surg Oncol 2018;25:1381-1394.

24. Amini B, Boyle SP, Boutin RD, Lenchik L. Approaches to Assessment of Muscle Mass and Myosteatosis on Computed Tomography: A Systematic Review. J Gerontol A Biol Sci Med Sci 2019;74:1671-1678.

25. O'Sullivan B, Davis AM, Turcotte R, Bell R, Catton C, Chabot P, Wunder J, Kandel R, Goddard K, Sadura A, Pater J, Zee B. Preoperative versus postoperative radiotherapy in soft-tissue sarcoma of the limbs: a randomised trial. Lancet 2002;359:2235-41.

26. Heymsfield SB, Wang Z, Baumgartner RN, Ross R. Human body composition: advances in models and methods. Annu Rev Nutr 1997;17:527-58.

27. Mourtzakis M, Prado CM, Lieffers JR, Reiman T, McCargar LJ, Baracos VE. A practical and precise approach to quantification of body composition in cancer patients using computed tomography images acquired during routine care. Appl Physiol Nutr Metab 2008;33:997-1006.

28. De Amorim Bernstein K, Bos SA, Veld J, LozanoCalderon SA, Torriani M, Bredella MA. Body composition predictors of therapy response in patients with primary extremity soft tissue sarcomas. Acta Radiol 2018;59:478-84. 